\title{
Prediction of esophageal varices in liver cirrhosis by transient elastography and aspartate aminotransferase - to - platelet ratio index (APRI)
}

\author{
Debashis Kumar Sarkar ${ }^{1 *}$, Golam Azam², Majharul Haque ${ }^{3}$, Anisur Rahman ${ }^{4}$
}

\begin{abstract}
Background: Cirrhosis is a chronic liver disease that can be caused by almost all progressive liver injuries, such as viral, autoimmune, hereditary, metabolic and toxin mediated liver diseases. Esophageal varix (EV) is a frequent complication of cirrhosis. Although the survival rate of patients with bleeding cirrhosis has improved because of the progress in variceal hemorrhage management, the hospital mortality rate is still around $14.5 \%$ cases. Early detection of EV in all patients with liver cirrhosis is required in order to reduce the mortality.
\end{abstract}

Method: This observational study was done at department of Gastrointestinal, Hepatobiliary \& Pancreatic Disorders (GHPD), BIRDEM General Hospital, Dhaka, during the period of August 2015 to October 2016. A total of 65 patients with cirrhosis of liver were included. Complete blood count, liver function test, endoscopy of upper GIT, ultrasonography, transient elastogram were done for all patients. Statistical analysis was done with SPSS version 22.

Result: The study included 65 cirrhotic patients, among them $66.2 \%$ were male. The mean age was 53.8 years. For predicting EVS, transient elastography at a cutoff value of $18 \mathrm{kpa}$ demonstrated a sensitivity was $88.7 \%(95 \%$ $C I=82.3-92.7)$, specificity $75.0 \%$ (95\% CI=46.9-92.6), PPV 94.0\% (95\% CI=87.2-98.2), NPV 60.0\% (95\% CI=37.5 -74.0), AUC was 0.769. In APRI for prediction of EVs at cutoff value 1.00, sensitivity was $63.3 \%(95 \%$ CI=55.6-65.4), specificity 83.3.0\% (95\% CI=53.7-97.0), PPV 94.3\% (95\% CI=84.1-99.0), $N P V 33.3 \%(95 \% C I=21.5-38.8)$ and AUC was 0.779 .

Conclusion: A significant positive correlation found between transient elastography with EVs in cirrhotic patients. Liver stiffness value at $18 \mathrm{Kpa}$ can predict EVs in cirrhotic patients. On contrary, APRI had a less(negative predictive value) NPV that showed there is no satisfactory cutoff value for APRI to be used as a predictor of EVs.

Key words: Liver cirrhosis, esophageal varices (EV), transient elastography, Aspartate aminotransferase-to-Platelet ratio index (APRI).

\section{Introduction:}

Cirrhosis of liver is a condition that has a variety of manifestation and complications, some of which can be life threatening (e.g. variceal bleeding). However it has become apparent that when the underlying insult (e.g. Chronic hepatitis $\mathrm{C}$, hemochromatosis) has been removed, reversal of

1. Medical Officer, ShaheedSuhrawardy Medical College, Dhaka

2. Associate Professor, Department of Gastrointestinal, Hepatobiliary and Pancreatic Disorders (GHPD), BIRDEM General Hospital, Shahbag, Dhaka,Bangladesh.

3. Junior consultant (Medicine), Narayangonj General Hospital, Narayangonj

4. Professor, Department of Gastrointestinal, Hepatobiliary and Pancreatic Disorders (GHPD), BIRDEM General Hospital, Shahbag, Dhaka, Bangladesh.

*Debashis Kumar Sarkar and GolamAzam had equal contributions and will be considered as principal authors.

\section{Corresponding Author:}

Golam Azam MBBS, MD (Hepatology).

Associate Professor, Department of Gastrointestinal, Hepatobiliary and Pancreatic Disorders (GHPD)

BIRDEM General Hospital, Shahbag, Dhaka,1000, Bangladesh

Email: drgolamazam@gmail.com fibrosis also seen. Portal hypertension is a significant complication of cirrhosis and is responsible for development of esophageal varices. ${ }^{1}$

Early detection of varices is important for treatment and prevention of progression. Over the last decade, it has become the common practice to screen known cirrhotics with endoscopy to look for esophageal varices. Several factors predict the risk of bleeding, including the severity of cirrhosis (Child's class, MELD score), the height wedged hepatic vein pressure, the size of varices, and some endoscopic stigmata, including red wale signs, haematocystic spots, diffuse erythema, bluish colour, cherry red spots or white nipple spots. ${ }^{2}$

There is no reliable noninvasive predictor for the presence or absence of esophageal varices that can be adopted in clinical practice. Various study show that transient elastogram and APRI for the assessment of oesophagealvarices in cirrhotic patient has a excellent accuracy. ${ }^{3}$

The parameter is chosen as it allows us to assess velocity of ultrasound wave through the fibrosed liver and another parameter like simple blood test aspartate aminotransferase and platelet count are easily obtainable and noninvasive and can be done as a routine liver function test. So, considering these results can be very helpful in predicting the esophageal varices. ${ }^{4}$ In our country most of the patients with cirrhosis present at advanced decompensated stage. Endoscopic 
evaluation for esophageal varices is not always possible at most of the health care centers. Though endoscopy is gold standard to find varices, in order to relieve the patients from discomfort and the risk of rupture during endoscopy and to reduce costs, efforts to find non invasive methods like transient elastography and Aspartate aminotransferase-to-Platelet ratio index (APRI) are thought to be promising.

About $80 \%$ of patients with cirrhosis of liver will eventually develop varices. ${ }^{5}$ In cirrhotic patients who do not have esophageal varices at initial endoscopy, new varices will develop at a rate of approximately $5 \%$ per year. Up to $25 \%$ of patients with newly diagnosed varices will experience variceal bleeding within two years. ${ }^{1}$ In patients with cirrhosis who are being followed chronically, the development of portal hypertension is usually revealed by the presence of thrombocytopenia, the appearance of an enlarged spleen, or the development of ascites, and/or esophageal varices with or without bleeding. ${ }^{2}$

Endoscopy is the only means to directly visualize varices which are a consequence of portral hypertension. ${ }^{6}$ The predisposing factors of bleeding are large size of the varices, endoscopic variceal features such as red spots and stripes, high portal pressure and liver failure. Drugs capable of causing mucosal erosion, such as salicylates and NSAIDs can also precipitate bleeding. ${ }^{7}$ Current practice guidelines recommended endoscopic screening for the presence of esophageal varices in all patients with cirrhosis. If varices are not present, screening endoscopy should be repeated 2-3 years or sooner if there is evidence of hepatic decompensation. ${ }^{5}$ Other methods for detection of varices are ultrasonogram with Doppler study, CT scan, Gadolium-enhanced MRI and endosonography ${ }^{1}$.

Several studies have recently attempted to identify non-invasive predictors of esophageal varices. They are platelet count, AST-to-ALT ratio, AST-to-platelet ratio index (APRI),Platelet count/ spleen diameter ratio, Lok index, Forns' index, Fib-4 and fibroindex. Of these Transient elastogram, APRI, and Platelet count/spleen ratio are promising predictors. ${ }^{8}$ It was suggested that liver stiffness measured by transient elastography, a novel non-invasive technology may reflect not only fibrosis and portal pressure but it may even predict the presence or absence of large esophageal varices, in patient with cirrhosis. Liver stiffness values significantly correlates with the grade of esophagealvarices. The liver stiffness value of $19.2 \mathrm{kPa}$ was highly predictive for the presence of esophageal varices ${ }^{6}$.

Ultrasound elastography offers a number of advantages. It is easy to use and inexpensive. The acquisition speed is very high(less than one tenth of a second peracquisition) and, therefore the acquisitions are not biased by cardiac and respiratory movements. Once the probe is positioned correctly, the measurement is fully automated and independent from the operator. ${ }^{9}$ Aspartate aminotransferase to platelet ratio index (APRI) was first described for the non-invasive predictor of fibrosis which is the major cause of portal hepertension in cirrhosis and platelet count on its dominator, a variable knowingly associated with the presence of esophageal varices. Patients with an index lower than this cut off were supposed not to have esophageal varices. APRI at a cut off 1.3 was used to predict the existence of oesophageal varices. ${ }^{10}$ Platelet count/spleen diameter ratio as measured by abdominal ultrasound could be an accurate predictor of esophageal varices.In patients with compensated cirrhosis, the higher the ratio, the less likely it is that a patient will have varices. During the course of cirrhosis repeated course of endoscopy is recommended. As this intervention is expensive and often poorly accepted by patients who may refuse further follow up, there is a need for non-invasive methods to predicts the progression of portal hypertension as well as the presence and size of esophageal varices. ${ }^{6}$ This study was aimed to assess the liver stiffness values by transient elastography for predicting esophageal varices in cirrhotic patients and to assess the value of APRI for predicting esophageal varices in cirrhotic patients.

\section{Materials and methods}

This study was an observational cross sectional study carried out at the department of Gastrointestinal, Hepatobiliary and Pancreatic Disorders (GHPD), BIRDEM General Hospital, Dhaka, Bangladesh during the period of August 2015 to October 2016. Adult patients age $\geq 18$ years who are suffering from cirrhosis irrespective of cause were included and the following type of patients were excluded from this study: Patient having active gastrointestinal bleeding at the time of admission, those who had known severe co-morbid disease, known case of hematological malignancy or bleeding disorders, patient who was morbidly obese and tense ascites and CLD patient who had history of variceal band ligation or sclerotheraphy. Demographic and clinical variables were age, sex,etiology of cirrhosis of liver, jaundice, ascites, hepatic encephalopathy, edema, spider, testicular atrophy, gynaecomastia. Laboratory variables were haemoglobin level, serum albumin, serum billirubin, AST, ALT, Prothrombin time, platelet count, Endoscopic variceal grade, Transient elastography score.

Endoscopy of upper GIT were done by Olympus GIF-Q 160 video endoscopy in endoscopy room in presence and under direct supervision of the thesis guide and co guide. Varices were classified into small medium \& large by WGO practice guideline grading system.

Liver stiffness status was performed via transient elastography. All reading are taken from right lobe of liver with patient lying at supine position and with right arm in full abduction, An appropriate site for liver stiffness acquisition was identified in the mid axillary line using ultrasound probe. The median value of 10 successful acquisition, express in $\mathrm{kPa}$, was kept as representative of the liver stiffness. ${ }^{11}$

APRI was measured by the following formula-

$\mathrm{APRI}=\frac{\mathrm{AST}(\text { times above upper limit of normal })}{\left(\text { Platelet } \mathrm{x}^{10} / \mathrm{L}\right)} \times 100$ 
After collection of information these data, checked, verified for consistency \& edited for finalized result. After editing \& coding, the coded data entered into the computer by using the SPSS (Statistical Package for Social Sciences) version-22.0 software. Data cleaning validation \&analysis performed using the SPSS software. Statistical analyses by using appropriate statistical tool like $t$-test and chi square test. The results were presented in tables in mean, standard deviation (SD) \& percentages. Statistical significance set at 0.05 level and confidence interval at $95 \%$ level. Ethical clearance was taken from the ethical review committee of Birdem General hospital (BADAS).

\section{Results:}

Total 65 patients with cirrhosis were enrolled in this study. Subjects of the study was male dominant. Abdominal distension was more common. Anaemia, Leukonychia, oedema and spider were frequently present.

Figure 1 shows the aetiology of liver cirrhosis. Most of the patients were non $\mathrm{B}$ and non $\mathrm{C}$ related.

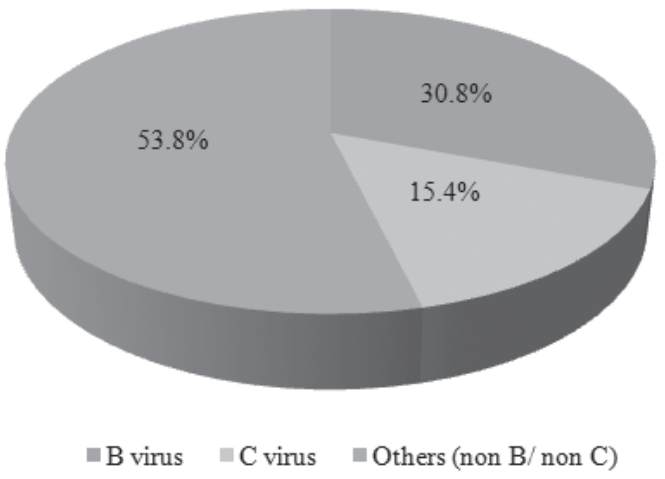

Fig1
Table 1

\begin{tabular}{ll}
\hline Parameters & n (\%) \\
\hline Age in years (Mean \pm SD) & $53.8 \pm 13.7$ \\
Gender & \\
$\quad$ Male & $43(66.2)$ \\
$\quad$ Female & $22(33.8)$
\end{tabular}

History

Hematemesis

$11(16.9)$

Melaena

Yellow coloration of sclera/urine (Jaundice)

Abdominal distention or swelling

Hepatic encephalopathy

\section{General examination findings}

Anaemia

Jaundice

Leuconychia

$39(60.0)$

Oedema

Gynaecomastia(among 43 male)

Spider

$30(46.2)$

\section{Abdominal examination}

Hepatomegaly

Splenomegaly

29 (44.6)

Ascites

Mild

Marked

Testicular atrophy(among 43 male)

Table 1: Shows demographic profile and clinical findings of the study populations $(n=65)$

Table-2. Shows comparison of patients with and without varices according to investigations $(n=65)$

Table 2

\begin{tabular}{llll}
\hline Investigations & $\begin{array}{l}\text { Present } \\
\text { (Mean } \pm \text { SD) }\end{array}$ & $\begin{array}{c}\text { Varices } \\
\text { Absent } \\
\text { (Mean } \pm \text { SD) }\end{array}$ & P value \\
\hline Platelet count (x109/L) & $101.01 \pm 37.74$ & $131.54 \pm 46.42$ & $0.018^{\#}$ \\
ALT (U/L) & $42.44 \pm 22.05$ & $51.58 \pm 52.89$ & $0.856^{\# \#}$ \\
AST (U/L) & $52.26 \pm 25.13$ & $33.75 \pm 11.31$ & $0.008^{\# \#}$ \\
INR & $1.00 \pm 0.15$ & $1.12 \pm 0.21$ & $0.023^{\#}$ \\
APRI & $1.58 \pm 1.20$ & $0.73 \pm 0.35$ & $0.003^{\# \#}$ \\
Transient Elastogram & $34.57 \pm 20.00$ & $13.32 \pm 10.63$ & $<0.001^{\# \#}$ \\
\hline
\end{tabular}

\#Mann-Whitney U test was done to measure the level of significance

\#Unpaired t test was done to measure the level of significance

Statistical significance was set at $\mathrm{p}<0.05$ and confidence interval set at $95 \%$ level. 
Table 3: Shows evaluation of different cutoff points for APRI as a predictor of esophageal varices in cirrhotic patients ( $\mathrm{n}=65$ ) APRI for prediction of EVs at cutoff value 1.00, sensitivity was 63.3\% (95\% CI=55.6-65.4), specificity $83.3 .0 \%$ (95\% $\mathrm{CI}=53.7-97.0)$, PPV 94.3\% (95\% CI=84.1-99.0), NPV 33.3\% (95\% CI=21.5-38.8).

Table 3

\begin{tabular}{lllll}
\hline Cutoff points for APRI & Sensitivity & Specificity & PPV & NPV \\
\hline 0.50 & $90.6(86.5-95.7)$ & $25.0(7.1-47.5)$ & $84.2(80.4-89.0)$ & $37.5(10.7-71.3)$ \\
0.90 & $67.9(61.6-73.4)$ & $58.3(30.4-82.4)$ & $87.8(79.6-94.9)$ & $29.2(15.2-41.2)$ \\
1.00 & $63.3(55.6-65.4)$ & $83.3(53.7-97.0)$ & $94.3(84.1-99.0)$ & $33.3(21.5-38.8)$ \\
1.30 & $49.1(42.5-50.8)$ & $91.7(62.7-99.6)$ & $96.3(83.4-99.8)$ & $28.9(19.8-31.4)$ \\
\hline
\end{tabular}

ROC curve shows test accurateness (Figure 2) of APRI for the prediction of esophageal varices $(\mathrm{AUC}=0.779$ and $\mathrm{p}=0.003)$

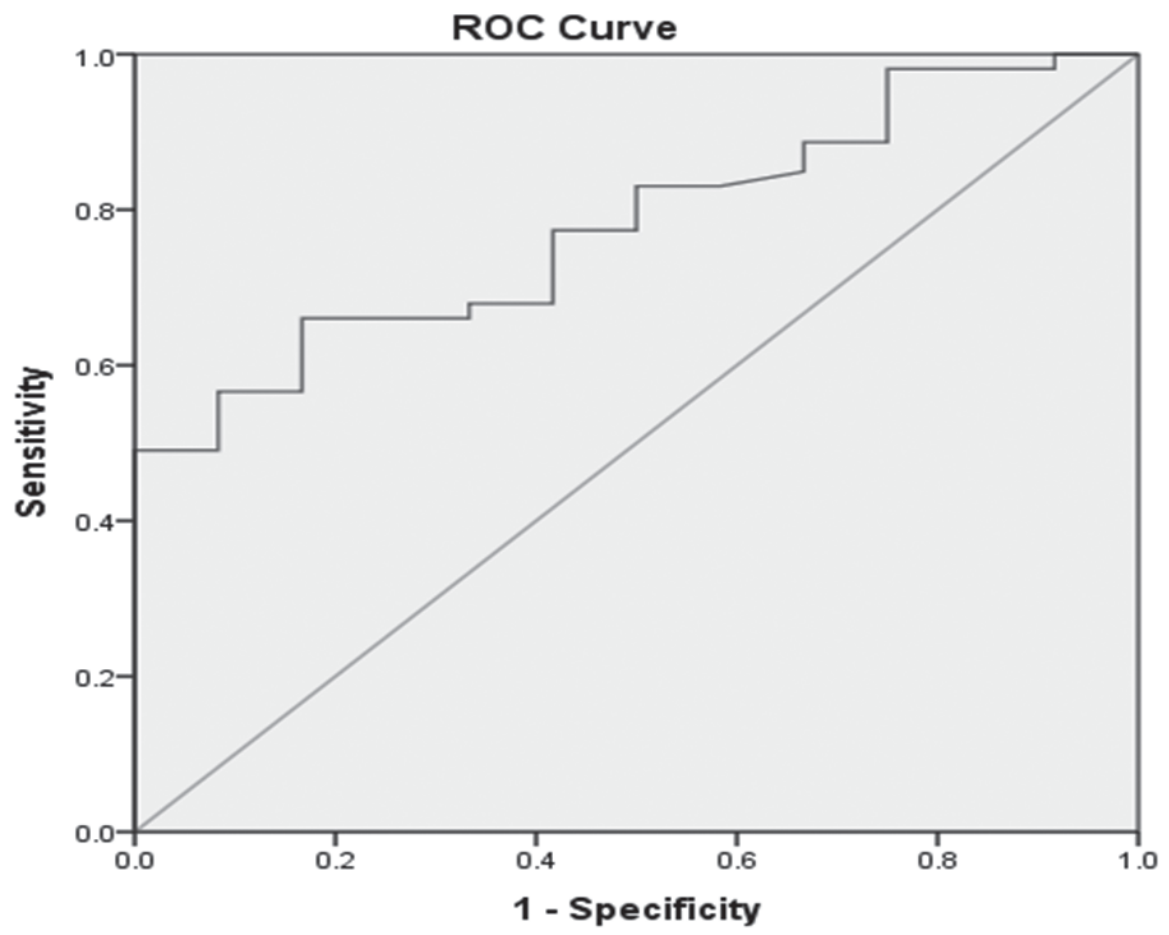

Diagonal segments are produced by ties.

Fig 2

Table 4: Shows evaluation of different cutoff points for Transient Elastogram Liver stiffness value as a predictor of esophageal varices in cirrhotic patients $(\mathrm{n}=65)$ transientelastography for predicting EVs, at a cutoff value of 18 kpa demonstrated a sensitivity was $88.7 \%(95 \% \mathrm{CI}=82.3-92.7)$, specificity $75.0 \%(95 \% \mathrm{CI}=46.9-92.6), \mathrm{PPV} 94.0 \%(95 \% \mathrm{CI}=87.2-98.2)$, NPV $60.0 \%(95 \% \mathrm{CI}=37.5-74.0)$.

Table 4

\begin{tabular}{lllll}
\hline Liver stiffness value (Kpa) & Sensitivity & Specificity & PPV & NPV \\
\hline 15 & $88.7(82.9-93.9)$ & $50.0(24.5-73.0)$ & $88.7(82.9-93.9)$ & $50.0(24.5-73.0)$ \\
18 & $88.7(82.3-92.7)$ & $75.0(46.9-92.6)$ & $94.0(87.2-98.2)$ & $60.0(37.5-74.0)$ \\
23 & $73.6(66.9-77.7)$ & $75.0(45.7-93.0)$ & $92.9(84.5-98.0)$ & $39.1(23.8-48.5)$ \\
26 & $64.2(57.5-68.2)$ & $75.0(45.4-93.1)$ & $91.9(82.3-97.8)$ & $32.1(19.5-39.9)$ \\
\hline
\end{tabular}


ROC curve shows test accurateness of Transient Elastogram Liver stiffness value for the prediction of esophageal varices $(\mathrm{AUC}=0.769$ and $\mathrm{p}=0.004)$ (Figure 3 )

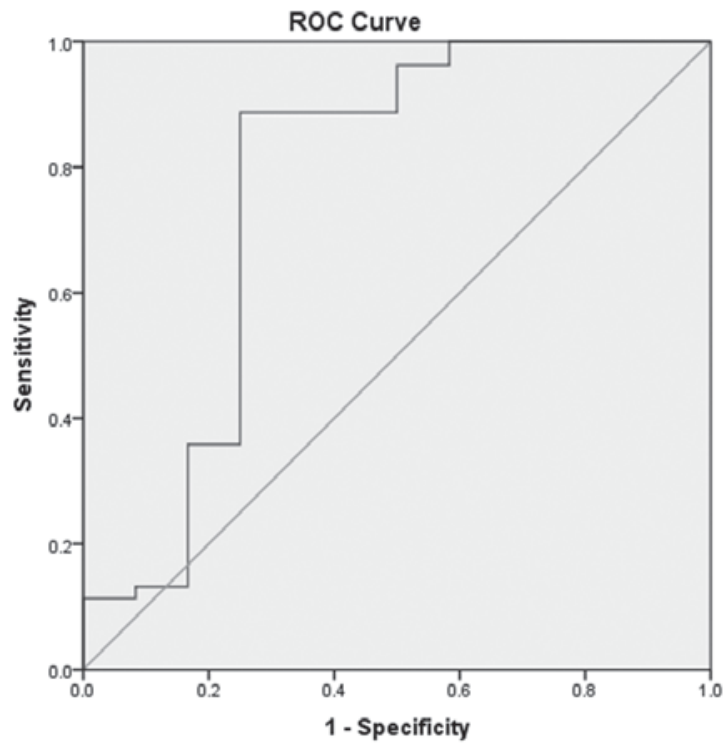

Fig 3

\section{Discussion:}

Cirrhosis is a common clinical problem in Bangladesh. Endoscopy in the gold standard for the diagnosis of varices screening with Endoscopy to identify EV in all cirrhotic patient at baseline as well as periodic intervals is recommended by current guidelines, Necessitating other easier modalities for diagnosis and monitoring of portal hypertension. Thus methods of predicting the presence of EVs noninvasively are in great demand to avoid unpleasant endoscopy and to improve the management. Several noninvasive methods have emerged in recent years by assessing simple laboratory, clinical and sonographic parameter such as splenomegaly, platelet count, portal vein diameter, AST, ALT, Transient elastogram. Giannini, Berzigotti found good indirect parameters for prediction of presence of EV in cirrhotic patients but available data in our country is limited..$^{12,13}$

This observational study was carried out with an aim to assess the liver stiffness value and aspartate aminotransferase (AST) with platelet count \& determine the state of esophageal varices by endoscopic evaluation. Finally our aim was to evaluate the relationship Liver stiffness value with esophageal varices \& APRI with varices in cirrhotic patient.

In this study, 65 patient was diagnosed as a cirrhosis. It was observed that cirrhosis were predominant at fifth decade 18 (27.7\%). The mean age of presentation (53.8\%) (table-1). Among them 43(66.2\%) were male \& 22(33.8\%) were female (table 1). In a study, Mattos found the mean age of cirrhosis is 56.7 years and male are predominant $59.7 \% .{ }^{10}$ The mean age and sex differences of above study correlate with our study.

Regarding clinical variables it was observed that 20 (30.8\%) had abdominal distension or swelling, 11(16.9\%) had hematemesis, $9(13.8 \%)$ had melena, $8(12.3 \%)$ had jaundice and $7(10.8 \%)$ had hepatic encephalopathy. Spider angioma and Gynaecomastia were observed in 30(46.2\%) and 13(20\%) respectively. On abdominal examination Hepatomegaly, splenomegaly, ascites, testicular atrophy were found in 6(9.2\%), 29(44.6\%), 23(35.4\%) \& 21(26.2\%) respectively. (table 1); Fraqueli observed 20\% had jaundice, $20 \%$ hematemesis, spenomegaly $35.7 \%$, and these observations are comparable with the current study. ${ }^{14}$

In our study, patient with EVs had lower platelet count $\left(\right.$ mean \pm SD $\left.101.01 \pm 37.7410^{9} / \mathrm{L}\right)$ than those without EVs $\left(\right.$ mean \pm SD $\left.131.54 \pm 46.4 \times 10^{9} / \mathrm{L}\right)$. This difference was statistically significant $(\mathrm{p}<0.035)$ among the groups. (Table 2). Sebastini found that the platelet count was lower in patient with esophageal varices (mean $\left.\pm \mathrm{SD}, 98.8 \pm 48.4 \times 10^{9} / \mathrm{L}\right)$ than patient without cirrhosis (mean $\pm \mathrm{SD}, 142.8 \pm 70.1 \mathrm{x} 10^{9} / \mathrm{L}$ ) which is almost similar to our results. ${ }^{8}$

Chalasani reported that platelet count can predict the significant esophageal varices. ${ }^{15}$ Our study showed Hepatitis $\mathrm{B}$ virus (HBV) was $30.8 \%$, Heptitis C virus was (HCV) $15.4 \%$ and other causes was $53.8 \%$ (Figure 1). Sebastini showed, HCV 55.1\%, alcohol 30.4\%, HBV 8.\% \& others were $36.1 \%$ which differs from our study probably due to prevalence of $\mathrm{HCV}, \mathrm{HBV}$ and alcoholism are more in western countries and most patients of our study in were tertiary diabetic care hospital had diabetic related fatty liver disease which leads to CLD. ${ }^{8}$

APRI by its two component AST \& Platelet has different cut off value for its sensitivity and specificity, Positive predictive value (PPV), negative predictive value (NPV) vary. In this study, when APRI at cut off 1.00 was used in order to predict the existence of EVs, it was found moderate sensitivity $63.3 \%$ $(95 \% \mathrm{CI}=55.6-65.4)$, a specificity $83.3 \%(95 \% \mathrm{CI}=53.7-97.0)$, PPV $94.3 \% \quad(\mathrm{CI}=84.1-99.0)$, but NPV $33.3 \% \quad(95 \%$ $\mathrm{CI}=21.5-38.8)$. Other cut off points were also tested, but none of them could reach a significantly better negative predictive value (table 3 ). Mattos showed at cut of value 1.3 a sensitivity $64.7 \%(0.56-0.86)$, specificity $72.7 \%(\mathrm{CI}=0.59-0.86)$, PPV of $86.5 \%(\mathrm{CI}=0.79-0.94)$, NPV of $43.2 \%(\mathrm{CL}=0.32-0.55)$ which is almost similar to our study. ${ }^{10}$

Castera proposed the cutoff of 1.3 for APRI as a predictor of $\mathrm{EV}$, where they found sensitivity $68 \%$, specificity $64 \%$, PPV $51 \%$, NPP $78 \%$, which is dissimilar from our study. ${ }^{16}$ That study was only on hepatitis $\mathrm{C}$ positive patient \& Child A grade were included. In our study all etiologies \& all grade including compensated and decompensated cirrhosis were included. Tafarel studied at a higher cut of point (1.64) and found it is significant. Here in this study higher cutoff value also tested, though good specificity and positive predictive value were found, sensitivity and NPV were disappointing. ${ }^{17}$ Wang proposed lower cut of value 0.77 as the optimal one to predict EVs with a better sensitivity $71 \%$ \& NPV $79 \%$, but in our study in lower value, specificity \& NPV were disappointing. ${ }^{18}$

In current study the diagnostic performance of Transient elastography (TE) was tested, as a noninvasive tool for prediction of EV (Table 4). There were different cutoff values for their prediction. In this study, if liver stiffness value cutoff 
point is $18 \mathrm{Kpa}$, sensitivity $88.7 \%$ (95\% CI 82.7-92.7), specificity $75.0 \%$ (95\% CI 46.9-92.6). PPV 94\%, NPV $60.0 \%$. AUROC $=0.769, \mathrm{P}=0.004$ were observed indicating moderately high level of significance (Figure 4). Hassan showed that TE could diagnose the EV at a cutoff value of $18.2 \mathrm{Kpa}$. Its sensitivity $80 \%$, specificity $72 \%$, PPV $84 \%$, \& NPV $67 \% .{ }^{19}$ This was almost similar to our study. Liu showed cutoff value at 18 with $91 \%$ sensitivity \& $63 \%$ specificity which comparable to our study. ${ }^{20}$

Kitson showed the higher cutoff value of $25 \mathrm{Kpa}$ to predict EVs with a sensitivity $71.9 \%$, specificity $58.1 \%$, PPV $88 \%$, NPV $88 \%$ which is dissimilar to our study. ${ }^{11}$ At higher cutoff value in our study NPN in 32 which was not significant. Fraquelli also showed similar result which correlates with our study. ${ }^{14} \mathrm{Qu}$ show a meta-analysis with a good sensitivity $84 \%$, specificity $68 \%$, positive likelihood ratio 2.58 , and negative likelihood ratio 0.24 , which is also comparable to our study. ${ }^{21}$

The transient elastography and APRI has also been examined by many authours in many countries and different cutoff of value was proposed but lack the generalized consistent results. In our study transient elastography can predict the EVs but APRI did not perform better for detection of EVs.

This study had several limitations, as the study population was selected from one hospital in Dhaka city, so that the results of the study may not reflect the exact picture of the country, Sample size was small, and grading of varices may be subject to inter-observer variability.

In conclusion, the identification of non-endoscopic and non-invasive methods that can accurately predict EVs in cirrhosis of liver has been addressed in recent years. There was a significant positive correlation found between transient elastography and EVs in cirrhotic patients. Liver stiffness value of $18 \mathrm{Kpa}$ can predict EVs in cirrhotic patients and a useful adjunct for clinicians in the management of cirrhotic patients But APRI had a wide range of cut of points that proves there is no satisfactory cutoff value for APRI to be used as a predictor of EVs. Variceal bleeding is a serious complication of cirrhosis; APRI must have an excellent negative predictive value to exclude EVs. However, the efficacy of this noninvasive diagnosis is not proven sufficient enough to replace the endoscopy. Further study with large sample size with prospective cohort studies are needed to validate its efficacy.

\section{References}

1 Shah VH \& Kamath, 2016. Portal hypertension and variceal bleeding. In: Feldman M, Friedman LS \&Brandt LJ(eds). Sleisenger and Fordtrans Gastrointestinal and Liver Disease. 10th ed. Philadelpia, Elsevier.

2 Bacon BR.2012. Cirrhosis and its Complication. In: Lango DL, Kasper DL,JameonJL,FauciAS,Hauser SL and Loscalzo J (eds), Harison's principle of internal medicine, McGraw.Hill, New York.

3 Giannini E, Botta F, Borro P, Risso D, Romagnoli P, Fasoi F et al. Platelet count/spleen diameter ratio :proposal and validation of a non invasive parameter to predict the presence of esophageal varices in patients with liver cirrhosis. Gut $2003 ; 52(8): 1200-1205$.
4 Reed E, Stanley A, Forrest E, Gillespi R, Dicson S. Transient elastography as a predictor of esophageal varices, a comparison with other non invasive markers. Gut 2014; 60 :244-45.

5 Grace ND \& Minor, MA 2012. Portal hypertension and esophageal variceal hemorrhage. In: Greenberger NJ, Blumer RS, Burakoff R (eds), Current diagnosis and treatment,Mc Grew Hill, New York.

6 Par G, Trosits A, Pakidi F, Szabo I, Czimer J. Transient elastography as a predictor of esophageal varices in patients with liver cirrhosis. J Orv Hetil 2014;155(7) :270-6.

7 Walker BR Colledge NR, Ralston SR and Penman ID (eds), 2014. Devidson's principle and practice of medicine. 22nd ed.Edinburgh, Elsevier.: 942-3.

8 Sebastiani G, Tempesta D, Fattovich G, Laurent C, Halfon P. Prediction of esophageal varices in hepatic cirrhosis by simple non-invasive markers: Results of a multicenter, large scale study. J Hepatol $2010 ; 53: 630-8$.

9 Goldis A, Ratiu I, Lazar D,Kaukaulas D. Trasientelastography as a predictive tool for decompensation and the occurrence of complications in liver cirrhosis. J Hepatol 2010;52:161.

10 Mattos AZ, Mattos AA, Vianna, FF, Muffkopf MI, Pereiralima JC. Platelet count/ spleen diameter ratio:analysis of its capacity as a predictor of the existence of esophageal varices. Arq Gastroenterol 2010;47:275-8.

11 Kitson MT, Robert SK, Colman JC, Paul E, Button P. Liver stiffness and the prediction of clinically significant portal hypertension and portal hypertensive complications, Scandinavian journal of gastroenterology $2015 ; 50: 462-469$.

12 Giannini EG, Savarino V. Predictors of pathological severity in non-alcoholic fatty liver disease: is East meeting West in the middle? J GastroenterolHepatol 2007; 22 :461-3.

13 Berzigotti A, Gilabart R, Abraldes JG, Nicolau C, Bru C. Significant portal hypertension and esophageal varices in patients with compensated liver cirrhosis. Am J Gastroenterol 2008 ;10(3):1159-67.

14 Fraquelli M, Giunta M, Prozzi R, Rigamontti C, Della valle S, Massironi S. et al. Feasibility and reproducibility of spleen stiffness measurement by transient elastographyand its role in combination with liver transient elastography for predicting the severity of chronic viral hepatitis.J Viral Hepat2014.;21:90-98.

15 Chalasani NI, Imperiale TF, Ismail A, Sood G, Carey M, Wilcox $\mathrm{CM}$ et al Predictors of large esophageal varices in patients with cirrhosis. Am J Gastroenterol. 1999;94(11):3285-91.

16 CasteraL, Vergniol j, Fouchar J, Le Bail B, Chanteloup E, Haaser M et al. Prospective comparison of ransientelastography, fibrotest,APRI and liver biopsy for assessment of fibrosis in chronic hepatitis C.Gastroenterology $2013 ; 128: 343-50$.

17 Tafarel JR, Tolentino LH, Correa LM, Bonita DR, Matin FS. Prediction of esophageal varices in hepatic cirrhosis by noninvasive markers. Eur JGastroenterolHepatol 2011;23:754-8.

18 Wang JH, Cruah SK, Lu SN, Hung CH, Chen CH. Transient elastography and simple blood markers in the diagnosis of esophageal varices for compensated patients with hepatitis B virus related cirrhosis. JGastroenterolHepatol 2012;27:1213-18.

19 Hassan EM, Omar DA, El BeshlaweyML, Abdo M, El Askary. Can transient elastography Fib-4, Forns index, and loc score can predict esophageal varices in $\mathrm{HCV}$ related cirrhotic patients? GastroenterolHepatol 2014;37(2):58-65.

20 Liu F, Li TH, Li C, Huang C, Zhang HS. Non invasive assessment of portal hypertension in patients with liver cirrhosis using fibroscan. Chin J Hepatol 2013; 21:840-4.

21 Qu Y, Li T, Ye Q, Zhang L, Wang L. A beginning or the End? AMeta-analysis to Assess the Diagnostic Accuracy of Transient elastography for the Prediction of Esophageal Varices, Saudi Journal of gastroenterology 2016;22(5):345-52. 